\title{
Le référentiel « cinq piliers de la maîtrise de l'information scientifique » : élaboration et construction de l'outil
}

Bernard Pochet, Nancy Durieux, Caroline Collette, Sara Decoster, Mélodie Dieudonné and Sandrina Vandenput

\section{(2) OpenEdition Journals}

Electronic version

URL: http://journals.openedition.org/ripes/2850

DOI: $10.4000 /$ ripes.2850

ISSN: 2076-8427

Publisher

Association internationale de pédagogie universitaire

\section{Electronic reference}

Bernard Pochet, Nancy Durieux, Caroline Collette, Sara Decoster, Mélodie Dieudonné and Sandrina Vandenput, "Le référentiel « cinq piliers de la maîtrise de l'information scientifique » : élaboration et construction de l'outil", Revue internationale de pédagogie de l'enseignement supérieur [Online], 37(2) I 2021, Online since 15 March 2021, connection on 18 March 2021. URL: http://

journals.openedition.org/ripes/2850 ; DOI: https://doi.org/10.4000/ripes.2850

This text was automatically generated on 18 March 2021.

Article L.111-1 du Code de la propriété intellectuelle. 


\title{
Le référentiel « cinq piliers de la maîtrise de l'information scientifique » : élaboration et construction de l'outil
}

\author{
Bernard Pochet, Nancy Durieux, Caroline Collette, Sara Decoster, Mélodie \\ Dieudonné and Sandrina Vandenput
}

\section{Introduction}

\subsection{Le concept d'Information Literacy}

1 Le concept d'Information Literacy date de la fin des années 80 et a beaucoup évolué depuis (Pochet, 2012).

2 En 1989, le Presidential Committee on Information Literacy de l'American Library Association annonçait une nouvelle didactique de l'accès à l'information. Il adoptait le néologisme Information Literacy et le présentait comme un ensemble de compétences qui permettent aux individus de reconnaître un besoin d'information et les rendent capables de localiser, évaluer et utiliser l'information nécessaire (American Library Association, 1989).

3 Une définition récente de l'Information Literacy Group de la UK Library and Information Association a fait évoluer le concept et le décrit comme « (...) the ability to think critically and make balanced judgements about any information we find and use (...) It empowers us as citizens to reach and express informed views and to engage fully with society" (CILIP Information Literacy Group, 2018, p.3). Cette évolution suppose l'extension progressive de la définition du concept d'Information Literacy à la vie de tous les jours, à la vie en société, à l'éducation, au travail et à la santé (CILIP Information Literacy Group, 2018).

Principalement depuis l'accroissement des usages du numérique (Michaut et Roche, 2017), le concept de maîtrise de l'information constitue une vraie problématique en 
pédagogie universitaire. Accéder, évaluer, comprendre et utiliser l'information scientifique figurent parmi les objectifs de l'enseignement universitaire (Pochet, 2012).

Il n'est pas aisé de traduire en français ce néologisme anglo-saxon (Chevillotte, 2005). Les traductions les plus fréquentes sont : «littérisme», "alphabétisation informationnelle ", "culture de l'information », " compétences informationnelles », " infocompétences ", « littératie ", «maitrise de l'information » ou encore " éducation à l'information" (Le Deuff, 2008). Au cours des années 2000, dans le monde francophone, l'expression « maîtrise de l'information » a été privilégiée (Horton, 2008; Mittermeyer et Quirion, 2003). Cette dernière a été retenue pour le référentiel présenté dans cet article.

\subsection{Le cadre de travail}

6 La maitrise de l'information est une préoccupation de longue date pour les membres de l'ULiège Library, bibliothèque de l'Université de Liège, Belgique (Pochet et Thirion, 1998; Pochet et al., 2015). Outre ses participations actives dans le paysage francophone belge, avec des rayonnements internationaux réguliers, l'ULiège Library dispense des cours obligatoires inscrits dans différents cursus et propose des séances de formation à l'intention des membres de son institution.

7 En 2012, suite à l'ampleur des missions confiées aux bibliothécaires-enseignants de l'ULiège Library, le groupe de travail InfoLit a été constitué. Composé des 18 membres qui donnent au moins un cours ou organisent des formations, les principaux objectifs du groupe sont de coordonner les différentes activités proposées, de veiller à leur qualité et à une meilleure cohérence.

8 Parce qu'ils souhaitaient formaliser les objectifs et les contenus de leurs formations dispensées à l'ULiège (contexte académique), les membres du groupe de travail ont décidé en 2015 d'élaborer un référentiel propre à l'ULiège Library.

\subsection{Le projet de référentiel}

9 Avec la création d'un nouveau référentiel, le souhait des membres du groupe de travail InfoLit était de mettre à disposition un guide pour l'élaboration, l'amélioration voire l'évaluation par chaque enseignant de cours et formations ayant pour objectif la maîtrise de l'information scientifique. Il devait être utilisable pour tous les niveaux d'étude et pour les différents secteurs de connaissance couverts à l'ULiège, à savoir les sciences humaines (SH), les sciences et techniques (ST) et les sciences de la santé (SS).

10 Cet article décrit l'élaboration de ce référentiel. Il présente successivement la méthode de travail et le référentiel. Il discute ensuite des difficultés rencontrées, de la place de l'attitude éthique et de l'esprit critique ainsi que des spécificités de l'outil.

\section{Méthode de travail}

\subsection{L'équipe}

Sur base volontaire, un sous-groupe de sept bibliothécaires-enseignants de l'ULiège Library - issus des trois secteurs de l'Université (SH, ST, SS) - a décidé d'élaborer 
ensemble le référentiel. Après une première réunion en juillet 2015, ce sous-groupe s'est réuni à 11 reprises avec une périodicité d'une fois tous les deux ou trois mois. La dernière réunion a eu lieu en mai 2017.

\subsection{Démarche d'élaboration du référentiel}

Afin d'identifier les savoirs, savoir-faire et attitudes nécessaires à la maîtrise de l'information scientifique, le travail a consisté en l'analyse de trois contenus distincts : les "compétences attendues" par des enseignants et chercheurs de l'ULiège, une analyse approfondie de huit référentiels et les tables des matières des cours dispensés par les sept membres du groupe de l'ULiège Library. enseignants et chercheurs des trois secteurs (SH, ST, SS) de l'ULiège. Chaque membre du groupe était chargé de récolter, auprès de cinq enseignants et chercheurs, une liste de cinq «compétences attendues» pour les étudiants de premier cycle et cinq "compétences attendues" pour les étudiants de deuxième cycle. Ces personnes ressources ont été sélectionnées et consultées sur base de l'intérêt qu'elles portaient à la maitrise de l'information scientifique, en veillant à ce que chacune des 11 facultés de l'université soit représentée par au moins deux personnes.

Huit référentiels concernant la maîtrise de l'information ont été sélectionnés sur base de leurs caractères récent et académique puis analysés (contenu, structure et organisation):

- Australian and New Zeeland Information Literacy Framework (Bundy, 2004)

16 - Framework for Information Literacy for Higher Education ACRL (Association of College and Research Libraries, 2015)

17 - Information literacy framework for Wales (Welsh Information Literacy Project, 2011))

18 - Maîtrise de l'information des étudiants avancés (master et doctorat) - Eléments pour une formation (Groupe de travail des rencontres FORMIST, 2006)

19 - National Information Literacy Framework Scotland (Irving, 2011)

Référentiel de compétences informationnelles. Pour réussir son parcours de formation dans les établissements d'enseignement supérieur (Cachard et al., 2012)

21 - Référentiel des compétences en culture informationnelle (Stalder et al., 2011)

22 - The Seven Pillars of Information Literacy - SCONUL (Bent et Stubbings, 2011)

23 L'analyse des référentiels a permis (i) de dégager la notion de piliers (Bent et Stubbings, 2011) qui représentent les objectifs d'apprentissages principaux et (ii) de structurer le 5PMIS dès le début de sa conception. La notion de progression entre différents niveaux pendant le parcours universitaire (Groupe de travail des rencontres FORMIST, 2006; Stalder et al., 2011) a également été retenue pour structurer le 5PMIS.

Des savoirs, savoir-faire et attitudes attendues ont également été listés à partir de la table des matières des cours dispensés à des étudiants de premier et de second cycle par les bibliothécaires-enseignants du groupe de travail.

25 Après un premier regroupement des items équivalents en provenance des trois sources, une liste de 220 items a été créée. Afin de les organiser, de les regrouper et d'identifier les éventuels éléments manquants, la liste des savoirs, savoir-faire et attitudes a été 
introduite dans une carte heuristique puis dans un tableau. Après plusieurs réorganisations, les principaux objectifs d'apprentissage ont été identifiés.

Pour chacun d'eux, des ressources - à comprendre dans un sens englobant et donc ne se limitant pas seulement à une seule composante telle que les savoirs (Tardif, 2006) ont été listées. Ces ressources regroupent ainsi les savoirs, savoir-faire et attitudes à mobiliser pour atteindre chacun des objectifs.

Elles ont été classées par niveaux de développement: 1) niveau novice, 2) niveau intermédiaire et 3) niveau compétent (séquence de niveaux de développement adaptée de Benner, 1982).

Au cours des 22 mois de travail, le groupe a fait un large usage d'outils collaboratifs en ligne (PmWiki, Framapad, Framacalc, Mind42, Google Doc et Google Sheet) pour constituer les différentes listes ainsi que pour structurer, organiser et rédiger les contenus.

\subsection{Relecture par des pairs}

Deux phases de relecture par des pairs ont été programmées. La première a débuté en octobre 2016 et a impliqué des membres internes à l'ULiège : les 18 membres du Groupe de Travail InfoLit de l'ULiège Library, les 32 enseignants et chercheurs sollicités au début de ce travail, les membres du Comité de Direction de l'ULiège Library et une experte de l'Institut de Formation et de Recherche en Enseignement supérieur de l'ULiège.

La deuxième phase de validation, avec le référentiel quasiment abouti, a été réalisée par quatre didacticiens internes à l'ULiège et deux externes (Université Libre de Bruxelles, Belgique et Université de Montréal, Canada). Elle a également été réalisée par plusieurs membres de la Commission Pédagogie de l'Association des Directeurs et Personnels de Direction des Bibliothèques universitaires et de la Documentation (ADBU-France). Cette seconde phase a permis des ajustements sensibles.

31 C'est lors d'une dernière réunion, en mai 2017, que le schéma définitif du référentiel a été arrêté avec le concours d'une enseignante du Département des Sciences de l'Éducation de l'ULiège. Les semaines qui ont suivi ont été nécessaires pour finaliser la présentation et la mise en forme, y compris la version en ligne du référentiel.

\section{Le référentiel}

Le référentiel est articulé autour de cinq principaux objectifs d'apprentissage, appelés cinq piliers : définir, accéder, évaluer, organiser et intégrer l'information scientifique (Figure 1). Cette structure en cinq piliers a déterminé le nom du référentiel : les « cinq piliers de la maitrise de l'information scientifique » ou 5PMIS. 
Figure 1. Structure du référentiel 5PMIS

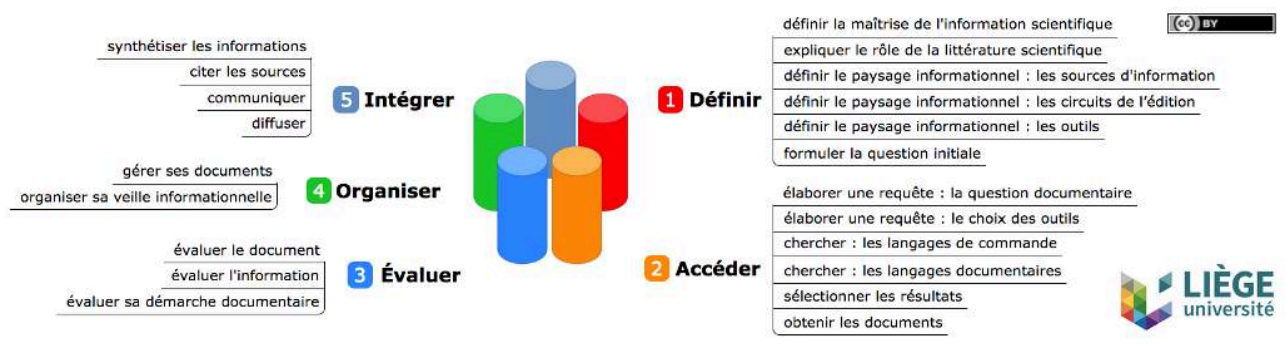

Bien que ces piliers soient numérotés de 1 à 5 , cela ne signifie pas qu'un pilier soit un prérequis d'apprentissage au pilier suivant, mais que la démarche informationnelle suit cette progression.

Les piliers couvrent 21 ressources principales (Figure 1), désignant comme défini cidessus les savoirs, savoir-faire et attitudes à mobiliser pour atteindre chacun des objectifs d'apprentissage.

Chacune de ces ressources est déclinée en ressources opérationnelles (Figure 2). Il y a au total 64 ressources opérationnelles. Elles ont été décrites avec précision en utilisant la taxonomie de Bloom (De Landsheere et De Landsheere, 1978). Les trois niveaux de développement (novice, intermédiaire et compétent) tiennent compte de la progression des étudiants au long de leur parcours universitaire. D'une discipline à l'autre, chaque niveau, indispensable prérequis du niveau suivant, peut être atteint à un moment différent du cursus.

Figure 2. Ressources opérationnelles pour la ressource 1.3

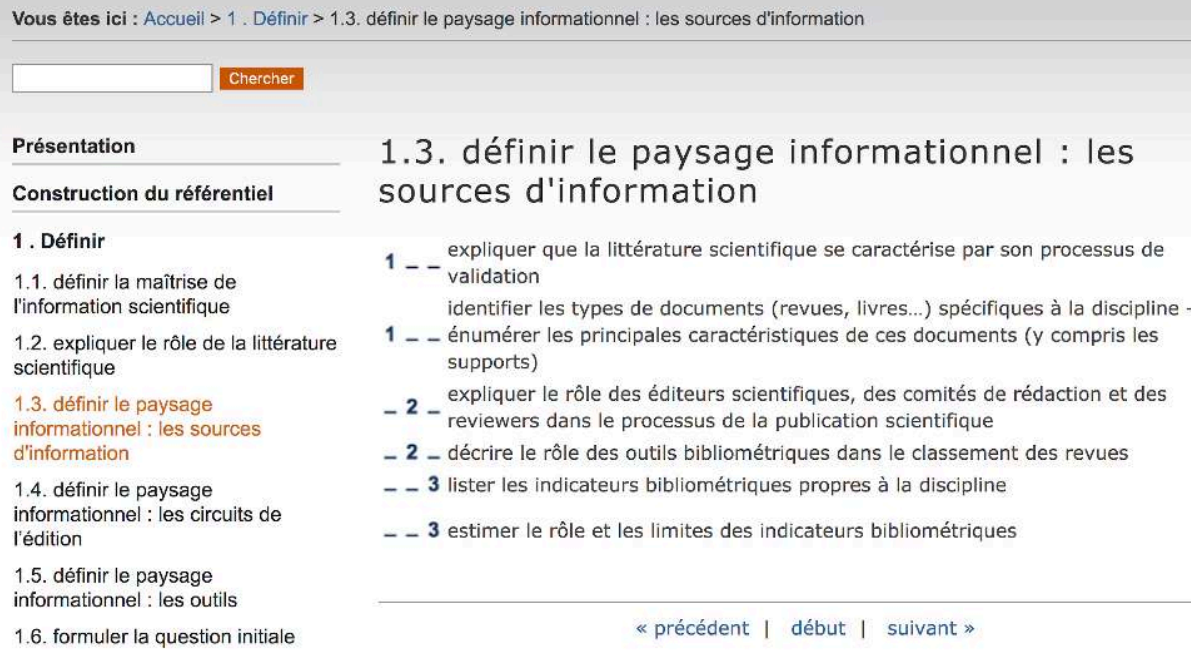

Présentation

Construction du référentiel

1. Définir

1.1. définir la maîtrise de l'information scientifique

1.2. expliquer le rôle de la littérature scientifique

1.3. définir le paysage

informationnel : les sources

d'information

1.4. définir le paysage

informationnel : les circuits de

l'édition

1.5. définir le paysage

informationnel : les outils

1.6. formuler la question initiale

1.3. définir le paysage informationnel : les sources d'information

1 expliquer que la littérature scientifique se caractérise par son processus de - validation

identifier les types de documents (revues, livres...) spécifiques à la discipline

1 _ - énumérer les principales caractéristiques de ces documents (y compris les supports)

_ 2 expliquer le rôle des éditeurs scientifiques, des comités de rédaction et des

- 2 - reviewers dans le processus de la publication scientifique

- $\mathbf{2}$ - décrire le rôle des outils bibliométriques dans le classement des revues

_ - 3 lister les indicateurs bibliométriques propres à la discipline

_ - 3 estimer le rôle et les limites des indicateurs bibliométriques

«précédent | début | suivant »

Le référentiel a été mis en ligne en Open Access (licence CC BY) en septembre 2017. Il est accessible à l'adresse https://infolit.be/5PMIS en version web et en version pdf.

La version web du référentiel permet deux modes d'accès. Le premier, à partir du bandeau supérieur ou à partir des menus (à gauche), suit la hiérarchie par pilier. Le second, en bas dans le menu, est organisé par niveau de développement (Figure 3). 
Figure 3. Entrée par niveau de développement. Exemple pour le niveau novice du deuxième pilier

\begin{tabular}{|c|c|}
\hline Présentation & Deuxième pilier : Accéder \\
\hline Construction du référentiel & 2.1. élaborer une requête : la question documentaire \\
\hline 1. Définir & - décomposer la question en concepts \\
\hline 2. Accéder & - organiser les concepts entre eux (logique booléenne) \\
\hline 3. Évaluer & $\begin{array}{l}\text { - délimiter le sens et le contenu de chaque concept afin d'éviter l'incertitude } \\
\text { terminologique }\end{array}$ \\
\hline 4. Organiser & 2.2. élaborer une requête : le choix des outils \\
\hline 5. Intégrer & $\begin{array}{l}\text { - identifier les différents outils de recherche documentaire/d'information pertinents pour } \\
\text { la question }\end{array}$ \\
\hline $\begin{array}{l}\text { Entrées par niveaux de } \\
\text { développement }\end{array}$ & $\begin{array}{l}\text { - démontrer les apports spécifiques de chaque outil de recherche } \\
\text { documentaire/d'information }\end{array}$ \\
\hline (1) Novice & 2.3. chercher : les langages de commande \\
\hline Premier pilier : Définir & $\begin{array}{l}\text { - employer les langages de commande (opérateur booléen, expression exacte, } \\
\text { troncature...) }\end{array}$ \\
\hline $\begin{array}{l}\text { Deuxième pilier : Accéder } \\
\text { Troisième pilier : Évaluer }\end{array}$ & - employer les fonctionnalités spécifiques aux outils (limites, facettes...) \\
\hline Quatrième pilier : Organiser & 2.4. chercher: les langages documentaires \\
\hline $\begin{array}{l}\text { Cinquième pilier : Intégrer } \\
\text { (2) Intermédiaire }\end{array}$ & $\begin{array}{l}\text { - expliquer la différence entre le langage documentaire contrôlé et le langage } \\
\text { documentaire libre }\end{array}$ \\
\hline (3) Compétent & - utiliser les langages documentaires \\
\hline Références bibliographiques & 2.5. sélectionner les résultats \\
\hline Autres référentiels & $\begin{array}{l}\text { question posée, en fonction de critères généraux (type de document, titre, résumé, } \\
\text { année) }\end{array}$ \\
\hline Crédits & 2.6. obtenir les documents \\
\hline Version i & $\begin{array}{l}\text { - identifier les différents moyens à disposition, avec leurs avantages et inconvénients, } \\
\text { pour obtenir un document }\end{array}$ \\
\hline Plan du site & $\begin{array}{l}\text { - utiliser, à bon escient, un ou plusieurs moyen(s) permettant d'obtenir le document } \\
\text { souhaité }\end{array}$ \\
\hline
\end{tabular}

\section{Discussion}

Cet article décrit un référentiel développé par des bibliothécaires-enseignants de l'ULiège Library concernant la maitrise de l'information. Ce référentiel cartographie les savoirs, savoir-faire et attitudes nécessaires à la recherche, à l'évaluation et à l'utilisation des publications issues de la recherche scientifique.

\subsection{Les difficultés rencontrées}

Le groupe a régulièrement éprouvé des difficultés à trouver un consensus autour des termes à employer (référentiel, compétences, savoirs, savoir-faire, socles, piliers, etc.). De nombreux allers-retours dans la littérature ont été nécessaires.

Le terme «compétences» a été celui qui a provoqué le plus de questionnements. Les définitions sont nombreuses et variées, parfois contradictoires, et mettent en évidence l'évolution du concept au fil des années (Perrenoud, 1995; Tardif, 2006). La définition de Tardif (2006, p. 22) a été choisie pour le développement du référentiel, à savoir qu'une compétence est «un savoir-agir complexe prenant appui sur la mobilisation et la combinaison efficaces d'une variété de ressources internes et externes à l'intérieur d'une famille de situations ». Le 5PMIS n'est donc pas un référentiel de compétences, mais un référentiel regroupant des ressources (savoirs, savoir-faire et attitudes) à prendre en compte pour l'élaboration de cours et de formations à la maitrise de l'information scientifique, sachant que ces ressources pourraient également être intégrées dans les référentiels de compétences existants ou en cours de rédaction à l'ULiège. 

une approche transversale qui permette d'arriver à des résultats qui soient pertinents et cohérents pour tous. Le niveau de précision dans la description des ressources a été systématiquement ajusté afin de rester dans une représentation commune aux trois secteurs (SH, ST, SS) de l'ULiège.

42

$$
\begin{aligned}
& \text { l'autre. N'ont été retenus que les points communs. Chacun doit interpréter les } \\
& \text { ressources en fonction de sa discipline. Par exemple, pour la ressource «identifier les } \\
& \text { types de documents (revues, livres...) spécifiques à la discipline - énumérer les } \\
& \text { principales caractéristiques de ces documents (y compris les supports) » du pilier 1, il } \\
& \text { est évident que les types de documents vont être bien différents en sciences } \\
& \text { biomédicales, en chimie ou en histoire contemporaine. Le contenu d'une formation } \\
& \text { tiendra compte des spécificités liées à la discipline. Cette ressource est néanmoins } \\
& \text { compréhensible pour les enseignants des trois secteurs. }
\end{aligned}
$$

\subsection{La place de l'attitude éthique et l'esprit critique dans le 5PMIS}

Le groupe de travail a longtemps cherché la meilleure manière d'intégrer les notions d'attitude éthique et d'esprit critique lors de la construction du référentiel. Il est en définitive apparu que l'attitude éthique et l'esprit critique, qui se déclinent dans l'ensemble du cursus universitaire, se devaient d'englober l'ensemble du référentiel. Il s'agit de ressources transversales qui constituent des postures indispensables dans la maîtrise de l'information scientifique.

De nombreuses ressources interviennent dans la construction de l'attitude éthique. Le suivi strict des normes de citation afin d'éviter le plagiat et la manipulation des données est un élément central des formations à la maitrise de l'information dans les milieux académiques (Bapte, 2019). Savoir identifier les différents types de sources, les processus de validation ou les méthodes d'accès sont également des éléments essentiels.

L'attitude éthique doit aussi être mobilisée lorsqu'il est question de bibliométrie, de coût de l'information, de choix d'outils documentaires ou d'Open Access. Ces concepts sont clairement identifiés dans le premier pilier et constituent une des spécificités du 5PMIS.

47 L'esprit critique (McCormick, 1983) intervient lui aussi à différentes étapes du processus de maitrise de l'information. Il doit être mobilisé lors de l'évaluation de la démarche de recherche d'information, lors de l'évaluation d'un document et de son contenu ainsi que lors de l'extraction des informations pertinentes.

\subsection{Les spécificités du $5 P M I S$}

Le référentiel 5PMIS se concentre sur la maîtrise de l'information essentielle à tous les étudiants-doctorants initiés à la recherche scientifique. Ainsi, les piliers du 5PMIS s'intègrent dans la démarche de la recherche scientifique. Cette spécificité est d'ailleurs à l'origine de l'adjectif « scientifique » dans le nom du référentiel.

49 Le côté multidisciplinaire et le classement des ressources en trois niveaux de développement sont également des spécificités du 5PMIS. Il a été conçu pour que

Revue internationale de pédagogie de l'enseignement supérieur, 37(2) | 2021 
chacun puisse se l'approprier, structurer son enseignement en fonction du public visé (premier cycle, deuxième cycle ou école doctorale) et de la discipline de celui-ci. Il n'est pas uniquement destiné aux professionnels de l'information et aux bibliothécaires. L'intérêt est qu'il puisse aussi être mobilisé dans des approches transversales, par des enseignants d'horizons divers.

C'est aussi un outil de communication et d'information. Tant au sein de l'ULiège qu'à l'extérieur, il pourra servir à informer les non spécialistes sur ce que font les formateurs en bibliothèque et sur ces compétences dites transversales à intégrer dans les référentiels de formation (Tardif et Dubois, 2013).

Le référentiel a été présenté à l'ULiège lors de la semaine de l'enseignement, du 21 au 24 novembre 2017 (Pochet et Thirion, 2017). Le référentiel doit être présenté dans les Facultés, en passant par les différents Conseils, les collègues, voire même les Autorités de l'Institution. Les membres du groupe de travail InfoLit de l'ULiège Library doivent jouer le rôle de relais au sein des Facultés avec lesquelles ils travaillent afin d'informer les enseignants sur les ressources qu'il pourrait être utile d'intégrer dans les référentiels de compétences (rédigés ou en cours de rédaction). Un autre objectif sera de susciter une collaboration interdisciplinaire entre les intervenants impliqués dans le développement de la maîtrise de l'information ainsi qu'une collaboration avec les étudiants afin de les conscientiser à l'importance de la problématique (Bélanger et al., 2017; Bélanger et Lemieux, 2017).

\section{BIBLIOGRAPHY}

Association of College and Research Libraries. (2015). Framework for information literacy for higher education. http://www.ala.org/acrl/sites/ala.org.acrl/files/content/issues/infolit/

framework1.pdf

American Library Association. (1989). Presidential committee on information literacy: Final report. http://www.ala.org/acrl/publications/whitepapers/presidential

Bélanger, G., Boisvert, D., Lemieux, M.-M. et Séguin, C. (2017). Qualité des pratiques de développement des compétences informationnelles au sein du réseau de l'Université du Québec [Rapport de recherche]. http://rapport-qualite-pdci.uquebec.ca/index.html?accueil.html

Bapte, V. D. (2019). Information literacy instruction determining the place of library professionals. DESIDOC Journal of Library \& Information Technology, 39(1), 39-46. https://doi.org/ 10.14429/djlit.39.1.13676

Bélanger, G. et Lemieux, M.-M. (2017). La collaboration au profit du développement des compétences informationnelles. Le tableau, 6, 5. http://pedagogie.uquebec.ca/portail/letableau

Benner, P. (1982). From novice to expert. American Journal of Nursing, 82, 402-407.

Bent, M. et Stubbings, R. (2011). The SCONUL seven pillars of information literacy. https:// www.sconul.ac.uk/sites/default/files/documents/coremodel.pdf 
Bundy, A. (dir.). (2004). Australian and New Zealand information literacy framework: Principles, standards and practice ( $2^{\mathrm{e}}$ éd.). Australian and New Zealand Institute for Information Literacy. https://www.utas.edu.au/__data/assets/pdf_file/0003/79068/anz-info-lit-policy.pdf

Cachard, P. Y. et al. (2012). Référentiel de compétences informationnelles. Pour réussir son parcours de formation dans les établissements d'enseignement supérieur. Association des directeurs et personnels de direction des bibliothèques universitaires et de la documentation (ADBU). http://adbu.fr/wpcontent/uploads/2013/02/Référentiel-ADBU-2012-165X235cm-3.pdf

Chevillotte, S. (2005). Voyage en infolit : Alice derrière l'écran ou quelques notes d'un voyage d'étude aux États-Unis. Bulletin des bibliothèques de France, 50(6), 64-70.

CILIP Information Literacy Group. (2018). CILIP definition of information literacy 2018. https:// infolit.org.uk/ILdefinitionCILIP2018.pdf

De Landsheere, V. et De Landsheere, G. (1978). Définir les objectifs de l'éducation. Thone.

Groupe de travail des rencontres FORMIST. (2006). Maîtrise de l'information des étudiants avancés (master et doctorat) : éléments pour une formation. https://www.enssib.fr/bibliotheque-numerique/ notices/21101-maitrise-de-l-information-des-etudiants-avances-master-et-doctorat-elementspour-une-formation

Horton, F. W. (2008). Introduction à la maîtrise de l'information. UNESCO. https:// unesdoc.unesco.org/ark:/48223/pf0000157020_fre

Irving, C. (2011). National information literacy framework (Scotland): Pioneering work to influence policy making or tinkering at the edges? Library Trends, 60(2), 419-438.

Le Deuff, O. (2008). La culture de l'information : quelles « littératies " pour quelles conceptions de l'information? Communication présentée au VIème colloque international du chapitre français de l'ISKO, Toulouse, France. https://archivesic.ccsd.cnrs.fr/sic_00286184/document

McCormick, M. (1983). Critical thinking and library instruction. Research Quarterly, 22(2), 341.

Michaut, C. et Roche, M. (2017). L'influence des usages numériques des étudiants sur la réussite universitaire. Revue internationale de pédagogie de l'enseignement supérieur, 33(1).

Mittermeyer, D. et Quirion, D. (2003). Étude sur les connaissances en recherche documentaire des étudiants entrant au 1er cycle dans les universités québécoises. https://www.enssib.fr/bibliothequenumerique/documents/1909-etude-sur-les-connaissances-en-recherche-documentaire-desetudiants-entrant-au-1er-cycle-dans-les-universites-quebecoises.pdf

Perrenoud, P. (1995). Des savoirs aux compétences : de quoi parle-t-on en parlant de compétences? Pédagogie collégiale, 9(1), 20-24.

Pochet, B. (2012). La littérature scientifique dans la formation des bioingénieurs à Gembloux : vingt années d'évolution du concept d'information literacy [thèse de doctorat, Université de Liège]. ORBi. http://hdl.handle.net/2268/135460

Pochet, B., Blondeel, S., Collette, C., Delbushaye, T., Deweer, L., Durieux, N. Frédéric, F., Lanners, C., Moreau, F. et Thirion, P. (éd.). (2015). Former aux compétences informationnelles à l'heure du Web 2.0 et des discovery tools : actes du colloque du 18 mai 2015. http://hdl.handle.net/2268/186578

Pochet, B. et Thirion, P. (1998). Le groupe formation des utilisateurs de l'Association belge de documentation : un bilan après plusieurs années de fonctionnement. Education et francophonie, 26(1), 9 . 
Pochet, B. et Thirion, P. (2017). Les bibliothèques et l'enseignement : le cas particulier de l'information literacy. Communication présentée à la Semaine de l'enseignement, Liège, Belgique. Repéré à http://hdl.handle.net/2268/216290

Stalder, P., Böller, N., Henkel, T., Landwehr-Sigg, S., Piccinini, S., Schubnell, B. et Stuber, B. (2011). Normes suisses sur les compétences en culture informationnelle. https:// www.informationskompetenz.ch/doc/e-lib/ 1_f_normes\%20suisses\%20sur\%20les\%20comptences.pdf

Tardif, J. (2006). L'évaluation des compétences : documenter le parcours de développement. Chenelière Éducation.

Tardif, J. et Dubois, B. (2013). De la nature des compétences transversales jusqu'à leur évaluation : une course à obstacles, souvent infranchissables. Revue française de linguistique appliquée, 18(1), 29-45. https://www.cairn.info/revue-francaise-de-linguistique-appliquee-2013-1-page-29.htm

Welsh Information Literacy Project. (2011). Information literacy framework for Wales: Finding and using information in 21st century Wales. https://libraries.wales/wp-content/uploads/2016/06/ Information_Literacy_Framework_Wales.pdf

\section{ABSTRACTS}

Scientific information literacy is a major concern for librarians involved in user training. At the University of Liège (Belgium), a framework has been created to map the knowledge, skills and attitudes necessary for the search, the evaluation and the use of publication from scientific research. Its purpose is to contribute to the development, improvement and even evaluation of courses and training aimed at mastering scientific information. This framework, called the "five pillars of scientific information literacy" (5PMIS), was developed on the basis of consultation of other frameworks for information literacy, the contribution of 32 teachers and researchers as well as the analysis of courses given at the University of Liège. After reviewing by internal or external members of the University of Liège, the framework was put online in September 2017. Tool for coherence and collaboration between the library-trainers of the ULiège Library, the 5PMIS covers the three knowledge sectors represented at the University, namely the human sciences, science and technology and the health sciences. It focuses on the academic context and takes into account different levels of development in the university curriculum.

The objective of this article is to describe the creation of this framework, designed so that each trainer can appropriate it according to the type of audience targeted. It is not only intended for information professionals and librarians. The advantage is that it can also be mobilized in transversal approaches, by teachers from different backgrounds. Although developed for the University of Liège, the 5PMIS can thus be used and adapted by other higher education institutions, both university and non-university.

La maîtrise de l'information scientifique est une préoccupation majeure des bibliothécaires impliqués dans la formation des usagers. À l'Université de Liège (Belgique), un référentiel a été créé afin de cartographier les savoirs, savoir-faire et attitudes nécessaires à la recherche, à l'évaluation et à l'utilisation des publications issues de la recherche scientifique. Il a pour finalité de contribuer à l'élaboration, l'amélioration voire l'évaluation, de cours et formations ayant pour objectif la maîtrise de l'information scientifique. Ce référentiel, appelé les «cinq piliers de la maîtrise de l'information scientifique " (5PMIS), a été développé à partir de la consultation d'autres référentiels, de l'avis de 32 enseignants et chercheurs ainsi que de l'analyse des cours dispensés à l'Université de Liège. Après relecture par des membres internes ou externes à 
l'Université de Liège, le référentiel a été mis en ligne en septembre 2017. Outil de cohérence et de collaboration entre les bibliothécaires-enseignants de l'ULiège Library, le 5PMIS couvre les trois secteurs de connaissance représentés à l'Université, à savoir les sciences humaines, les sciences et techniques et les sciences de la santé. Il se concentre sur le contexte académique et prend en compte différents niveaux de développement dans le parcours universitaire.

L'objectif de cet article est de décrire la création de ce référentiel, conçu pour que tout formateur puisse se l'approprier en fonction du type de public visé. Il n'est pas uniquement destiné aux professionnels de l'information et aux bibliothécaires. L'intérêt est qu'il puisse aussi être mobilisé dans des approches transversales, par des enseignants d'horizons divers. Bien que développé pour l'Université de Liège, le 5PMIS peut ainsi être utilisé et adapté par d'autres établissements de l'enseignement supérieur, universitaire ou non universitaire.

INDEX

Mots-clés: référentiel, information literacy, bibliothèque universitaire, formation, enseignement, communication

\section{AUTHORS}

\section{BERNARD POCHET}

Université de Liège, ULiège Library, Liège, Belgique, bernard.pochet@uliege.be

\section{NANCY DURIEUX}

Université de Liège, ULiège Library, Liège, Belgique, Nancy.Durieux@uliege.be

\section{CAROLINE COLLETTE}

Université de Liège, ULiège Library, Liège, Belgique, c.collette@uliege.be

\section{SARA DECOSTER}

Université de Leuven, Leuven, Belgique, sara.decoster@kuleuven.be

MÉLODIE DIEUDONNÉ

Université de Liège, ULiège Library, Liège, Belgique, melodie.dieudonne@uliege.be

\section{SANDRINA VANDENPUT}

Université de Liège, ULiège Library, Liège, Belgique, S.Vandenput@uliege.be 